\title{
$k$-Layer Straightline Crossing Minimization by Speeding Up Sifting
}

\author{
Wolfgang Günther ${ }^{1}$, Robby Schönfeld ${ }^{2}$, Bernd Becker ${ }^{1}$, and Paul Molitor ${ }^{2}$ \\ 1 Institute for Computer Science \\ Albert-Ludwigs-University, Freiburg, Germany \\ \{guenther, becker\}@informatik.uni-freiburg.de \\ 2 Institute for Computer Science \\ Martin-Luther-University, Halle-Wittenberg, Germany \\ \{schoenfe,molitor\}@informatik.uni-halle.de
}

\begin{abstract}
Recently, a technique called sifting has been proposed for $k$ layer straightline crossing minimization. This approach outperforms the traditional layer by layer sweep based heuristics by far when applied to $k$-layered graphs with $k \geq 3$. In this paper, we present two methods to speed up sifting. First, it is shown how the crossing matrix can be computed and updated efficiently. Then, we study lower bounds which can be incorporated in the sifting algorithm, allowing to prune large parts of the search space. Experimental results show that it is possible to speed up sifting by more than a factor of 20 using the new methods.
\end{abstract}

\section{Introduction}

Graphs are commonly used to represent information in many fields such as software engineering, project management, and social sciences. A good visualization of this information is necessary to get general ideas of relations. Minimizing the number of crossings in a drawing is a key problem, since crossings make it difficult to interpret a graph.

The $k$-layer straightline crossing minimization problem is the problem to minimize the number of crossings if the vertices are placed in $k$ layers (i.e. lines) and there are only edges between vertices of different layers. Vertices within each layer can be permuted in order to minimize the crossing number. Unfortunately, the problem is NP-hard even for two layers and a fixed order of the vertices of the first layer [3]. Many heuristics have been proposed in literature (for an overview, see [4,5]).

Recently, a new heuristic for $k$-layer straightline crossing minimization has been proposed [6]. This heuristic is based on a technique called sifting [10 which is usually applied to minimize Binary Decision Diagrams (BDDs) [1] that are widely used to model Boolean functions in formal logic verification and logic synthesis. The experiments made in [6] prove sifting to be very efficient, outperforming the heuristics known from literature for one-sided and $k$-layered straightline crossing minimization with $k \geq 3$. The drawback of this new heuristic is that its runtime is higher than the runtime of the best heuristics known from literature.

A similar problem is known for sifting applied to its original application. The size of BDDs largely depends on the underlying variable ordering 11. Sifting dynamically optimizes the variable ordering by finding the optimal position for each variable while keeping the relative order of all other variables. Although

J. Marks (Ed.): GD 2000, LNCS 1984, pp. 253258 2001.

(C) Springer-Verlag Berlin Heidelberg 2001 
sifting can be implemented efficiently, in many applications more than $90 \%$ of the CPU time is spent for variable reordering. Therefore, recently the use of lower bounds to speed up sifting has been proposed [2]: moving a variable is stopped if a lower bound on the resulting BDD size is larger than the best size found so far. In experiments it turned out that sifting can be sped up by $70 \%$ on average.

In this paper we propose the use of lower bounds for $k$-layer straightline crossing minimization using sifting. First, we show how the computation of the crossing matrix, which plays a key role in the algorithm, can be realized efficiently. Then, we formally prove lower bounds for the crossing number and we show how these lower bounds can be applied during sifting to prune parts of the search space which do not contain good solutions. We give experimental results showing that the sifting algorithm can be sped up by a factor of ten compared to the original algorithm of [6] by using the efficient computation of the crossing matrix. Using the lower bounds approach, sifting can be further sped up by a factor of 3. This clearly demonstrates the efficiency of the approach.

\section{Preliminaries}

\section{$2.1 k$-Layer Crossing Minimization}

A $k$-layered network is a directed graph $G=(V, E)$ whose vertex set $V$ is partitioned into $k$ subsets $V_{1}, \ldots, V_{k}$, i.e., $V_{1} \cup \ldots \cup V_{k}=V$ and $(\forall i \neq j) V_{i} \cap V_{j}=\emptyset$. Set $E$ only consists of edges which connect vertices of different levels $V_{i}$ and $V_{j}$ with $i<j . V_{i}$ is called the $i$-th layer of the graph.

We assume that graph $G$ is proper, i.e. that there are only edges between nodes of adjacent levels $V_{i}$ and $V_{i+1}$. Thus, permuting the vertices in a layer $V_{i}$, $i \in\{1,2, \ldots, k\}$, only affects crossings with adjacent layers.

The problem we have to solve is to find for all layers $i \in\{1, \ldots, k\}$ a permutation $\pi_{i}$ of the vertices of layer $V_{i}$ such that the number of edge crossings is minimized. Note that the number of edge crossings only depends on the permutations of the vertices and not on the exact positions of the vertices because edges are drawn as straight lines.

\subsection{Sifting for Crossing Minimization}

First, we only consider the one sided crossing minimization problem in order to introduce the basic sifting procedure. Assume that $k$ equals 2 and the permutation $\pi_{1}$ of layer $V_{1}$ is fixed. Then, the sifting algorithm [6] successively considers all vertices of $V_{2}$. For a vertex $v$ under consideration, the goal is to find a position for $v$ on the second layer with a minimal number of crossings, assuming that the relative order of all the other vertices remains unchanged. It is shown in [6] that the order in which the vertices are considered does not have much influence on the quality of the result.

In order to find the best position of a vertex, it is moved to all positions of its layer. This is done in three steps:

1. The vertex is exchanged with its successors until it is the rightmost vertex.

2. The vertex is exchanged with its predecessors until it is the leftmost vertex.

3. The vertex is moved back to the closest position which has led to the minimal number of crossings. 
Sifting can be extended to $k$-layer straightline crossing minimization using the following global sifting heuristic: In a first step, all vertices of the graph are sorted according to their degrees. We will denote this order by $\phi$ in the following. Now, each vertex is sifted within its layer, starting with the vertex with maximum degree. If no improvement is made, the Boolean variable fail is set to 1 and the vertices are sifted again, but starting with the vertex having minimum degree. Otherwise, the vertices are sifted again using the same order. As long as the value of the Boolean variable fail equals 0 , the order $\phi$ is reversed and the last two steps are repeated.

\subsection{Exchanging Neighboring Vertices}

Let us assume that the order of all layers but layer $i$ is fixed. In order to consider the effect of exchanging neighboring vertices in more detail, we have to introduce some new notations. We denote the set of edges incident with vertex $v$ by $E(v)$. Furthermore, the variables

$$
\delta_{u v}^{i}=\left\{\begin{array}{l}
1 \pi_{i}(u)<\pi_{i}(v) \\
0 \text { otherwise }
\end{array}\right.
$$

characterize the permutation $\pi_{i}$ of the $i$ th layer. $\delta_{u v}^{i}$ equals 1 if and only if vertex $u$ is placed before vertex $v$ in the ordering $\pi_{i}$. For each pair of nodes $u, v \in V_{i}$ with $u \neq v$, the crossing number $c_{u v}$ is defined to be the number of crossings between $E(u)$ and $E(v)$ if $\pi_{i}(u)<\pi_{i}(v)$ holds. Using these notations, for a given permutation $\pi_{i}$ the number of crossings between layers $i-1, i$, and $i+1$ is

$$
C\left(\pi_{i}\right)=\sum_{u \in V_{i}} \sum_{v \in V_{i}} \delta_{u v}^{i} c_{u v} .
$$

\section{Crossing Matrix}

In order to speed up the computation of the number of crossings in each step, we introduce a three-dimensional crossing matrix $c\left[i, p_{1}, p_{2}\right]$ which only requires update operations after interchanging two vertices. An entry $c\left[i, p_{1}, p_{2}\right]$ is set to $c_{\pi_{i}^{-1}\left(p_{1}\right), \pi_{i}^{-1}\left(p_{2}\right)}$ with respect to layer $i$ and it contains the number of crossings that edges incident to vertex $\pi_{i}^{-1}\left(p_{1}\right)$ have with edges incident to vertex $\pi_{i}^{-1}\left(p_{2}\right)$ if $p_{1}<p_{2}$ holds.

Now assume that we swap two vertices $u$ and $v$ of layer $i$ with $p_{1}=\pi_{i}(u)$, $p_{2}=\pi_{i}(v)$. Thus, all entries of the crossing matrix for layer $i$ and column $p_{1}$ have to be interchanged with the entries from column $p_{2}$. Analogously, all entries of row $p_{1}$ of layer $i$ have to be interchanged with row $p_{2}$ of layer $i$. Note, that we only operate on the crossing matrix and do not need to consider the graph itself. This swap operation has to be called each time two vertices are swapped.

When the vertex under consideration is set to its locally optimal position, some entries of the crossing matrix which belong to the neighboring layers $i-1$ and $i+1$ have to be updated, too. If vertex $v$ is moved from its original position $p_{1}$ to position $p_{\text {opt }}$, some entries in the crossing matrix referring to the neighboring layers $i^{\prime} \in\{i-1, i+1\}$ are also affected: entries of vertices adjacent to $v$ or adjacent to one of the vertices $\pi_{i}^{-1}\left(p_{1}\right), \ldots, \pi_{i}^{-1}\left(p_{\text {opt }}-1\right)$ for case $p_{1}<p_{\text {opt }}$ or $\pi_{i}^{-1}\left(p_{\text {opt }}+1\right), \ldots, \pi_{i}^{-1}\left(p_{1}\right)$ for case $p_{1}>p_{\text {opt }}$ have to be modified. Figure 1 describes the update procedure for the case $p_{1}<p_{\text {opt }}$ and layer $i^{\prime}=i-1$ in more detail. The case $p_{1}>p_{\text {opt }}$ is handled analogously. 

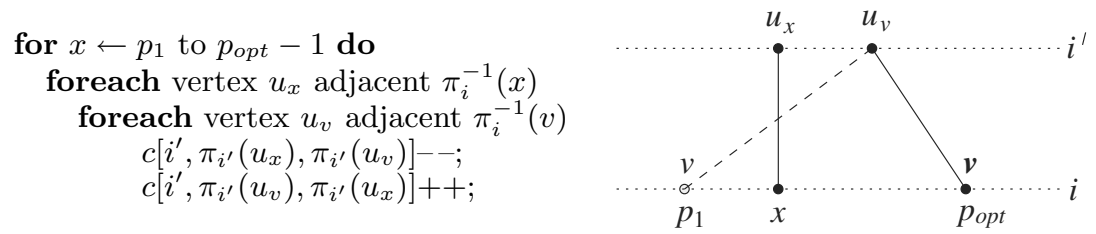

Fig. 1. The update operation for $p_{1}<p_{\text {opt }}$ and $i^{\prime}=i-1$.

\section{Lower Bound Sifting}

When sifting one vertex, the order of one layer $i$ is modified and the orders of the vertices of all the other layers remain unchanged. In this section, we give lower bounds on the resulting number of crossings and show how to use them during sifting.

In [4, a lower bound on the number of crossings for the one-sided crossing minimization is given:

$$
L B:=\sum_{u=1}^{\left|V_{i}\right|-1} \sum_{v=u+1}^{\left|V_{i}\right|} \min \left\{c_{u v}, c_{v u}\right\}
$$

is a lower bound on the number of crossings if permutation $\pi_{i}$ can be chosen arbitrarily and all other permutations are constant. In the following we show how this lower bound can be improved if only the position of one node of $V_{i}$ can be modified and the relative order of all other nodes remains the same.

A lower bound for moving one vertex $v$ in $\pi_{i}$ to the right while keeping the relative order of all other vertices unchanged is the following:

$$
\begin{aligned}
L B \rightarrow(v) & :=\sum_{\substack{u_{1}=1 \\
u_{1} \neq v}}^{\left|V_{i}\right|} \sum_{\substack{u_{2}=1 \\
u_{2} \neq v}}^{\left|V_{i}\right|} \delta_{u_{1} u_{2}}^{i} c_{u_{1} u_{2}}+\sum_{u=1}^{\left|V_{i}\right|} \delta_{u v}^{i} c_{u v}+\sum_{u=1}^{\left|V_{i}\right|} \delta_{v u}^{i} \min \left\{c_{v u}, c_{u v}\right\} \\
& =C\left(\pi_{i}\right)-\sum_{u=1}^{\left|V_{i}\right|} \delta_{v u}^{i} \max \left\{0, c_{v u}-c_{u v}\right\}
\end{aligned}
$$

Informally, this means that only those crossings are affected by moving vertex $v$ to the right in which an edge incident to $v$ and an edge incident to some node $u$ on the right of $v$ is involved. A similar lower bound can be used when moving a vertex to the left.

During sifting, moving a vertex $v$ can be stopped if it cannot improve the order already computed. To decide this, these lower bounds can be used. A sketch of the resulting algorithm moving a vertex rightwards is the following:

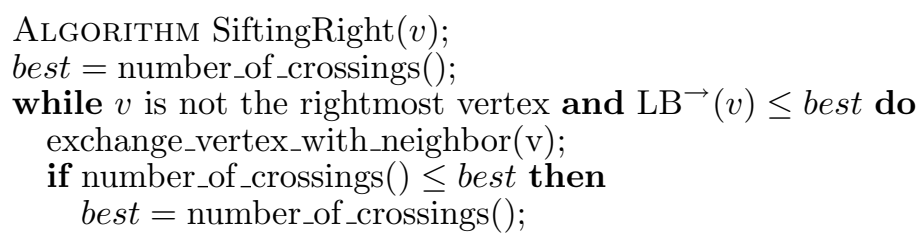

If the initial number of crossings is given, the lower bound for a vertex $v$ of the $i$ th layer can be computed in time $O\left(\left|V_{i}\right|\right)$. It can be updated in constant time. Thus, computing lower bounds is inexpensive. 
Table 1. Runtime requirements on sparse $4 \times n$ graphs.

\begin{tabular}{|c|c|c|c|c|c|c|c|c|}
\hline$n$ & \multicolumn{4}{|c|}{\begin{tabular}{|c|} 
runtime $[\mathrm{sec}]$ \\
siftingI siftingII siftingLB barycenter
\end{tabular}} & \multirow{2}{*}{\begin{tabular}{|c|}
$\begin{array}{c}\text { runtime }[\mathrm{sec}] \\
\text { combined }\end{array}$ \\
0.4
\end{tabular}} & \multicolumn{3}{|c|}{$\begin{array}{c}\text { quality }[\%] \\
\text { combined siftingLB barycenter }\end{array}$} \\
\hline 50 & \begin{tabular}{|l|}
21.7 \\
\end{tabular} & 1.7 & 0.6 & 0.01 & & 100.0 & 110.1 & 123.2 \\
\hline 60 & 29.6 & 3.0 & 1.1 & 0.01 & 0.8 & 100.0 & 112.3 & 123.5 \\
\hline 70 & 50.5 & 5.2 & 1.9 & 0.02 & 1.2 & 100.0 & 110.9 & 122.5 \\
\hline 80 & 80.2 & 8.6 & 3.1 & 0.02 & 2.0 & 100.0 & 110.3 & 122.9 \\
\hline 90 & 126.1 & 12.9 & 4.6 & 0.03 & 3.0 & 100.0 & 110.3 & 122.9 \\
\hline 100 & 200.8 & 20.3 & 7.2 & 0.03 & 4.8 & 100.0 & 111.3 & 123.1 \\
\hline
\end{tabular}

Note that using lower bounds does not affect the quality of the procedure, which is measured in number of crossings, since the positions which are not considered cannot be locally optimal. However, the final result can be different, since the optimal position of one node is not unique. Using lower bounds, a different (optimal) position can be chosen for a node, which may affect the result of the following nodes. Practically, experimental runs on random generated graphs of different size and density result in an average deviation of quality by at most 0.3 percent.

\section{$5 \quad$ Experimental Results}

The implementations of sifting are using algorithms form the AGD library 8 which is based on LEDA [7. The experiments have been carried out on a Sun UltraSPARC-IIi $\square$. All runtimes are given in CPU seconds.

Since the quality of the global sifting algorithm of [6] is about $20 \%$ better than the layer by layer based heuristics known from literature [4, in the following we only focus on runtime. It is shown that the algorithm can be sped up tremendously while the quality does not change significantly.

The implementation of the global sifting that was presented in [6] is denoted by sifting $\mathrm{I}$. The algorithm using the crossing matrix approach of Section 3 is named sifting II. Finally, algorithm sifting LB combines sifting II with the lower bound described in Section 4 .

In order to get an impression of the time savings we compare the three sifting algorithms in terms of run time requirement. In these experiments, we consider sparse $k \times n$-graphs, i.e., $k$-layered graphs $G_{k}=\left(V_{1} \cup \ldots \cup V_{k}, E\right)$ with $\left|V_{i}\right|=n$ for all $1 \leq i \leq k$ and $|E|=2 \cdot(k-1) \cdot n$.

In the experimental run we consider in this paper, only the number of vertices is varied. Table 1 (left part) shows the results for the three sifting algorithms for sparse $k \times n$-graphs with $k=4$ and $50 \leq n \leq 100$ taken over 100 samples. The first column gives the number $n$ of vertices per layer, the second, third, and fourth column show the runtime of sifting I, sifting II, and sifting LB in seconds, respectively.

It can be seen that the improved implementation for the crossing matrix is much faster than the original method. In some cases, the runtime for sifting II is even up to $90 \%$ less than for sifting I. Using lower bounds, the runtime can be further reduced by more than $65 \%$. Altogether, this is a speed-up of more than a factor of 20 .

However when considering runtime requirement the siftingLB approach continues to be dominated by common layer by layer sweep based heuristics of which barycenter is the most efficient method. It can be seen (Column 5 of Table 1) that

\footnotetext{
${ }^{1} 440 \mathrm{MHz}$ CPU, $256 \mathrm{MB}$ RAM.
} 
barycenter consumes practically constant computation time while the number of vertices per layer is being increased up to 100 .

The performance of lower bound sifting can be further improved by taking advantage of the computations of the barycenter method. Using the permutation of vertices obtained by barycenter as initial ordering for our sifting LB heuristic has two major effects: the number of crossings is usually reduced further and the application of the lower bound is more efficient as most vertices are already located in their locally optimal positions when starting with a good initial order in each layer. Column 6 of Table 1 (right part) shows the runtime of the combined heuristics barycenter and sifting LB. The computation effort could be reduced by nearly $40 \%$ in comparison with the single sifting LB heuristic. The relative numbers of crossings using sifting LB and barycenter compared to the combined method are given in the last three columns. It can be seen that for the combined method also the quality improves by $10 \%$ compared to sifting LB and by $20 \%$ compared to barycenter.

\section{Conclusions}

In this paper, we presented two improvements to the global sifting approach of 6 for $k$-layer straightline crossing minimization. First, a three-dimensional crossing matrix was proposed. Furthermore, lower bounds have been used to speed up sifting by determining parts of the search space which do not have to be considered. Experimental results showed that it is possible to reduce the runtime by more than a factor of 20 , without a loss of quality.

It is focus of current work to transfer other techniques known from BDD minimization like group sifting [9] to $k$-layer straightline crossing minimization.

\section{References}

1. R.E. Bryant. Graph - based algorithms for Boolean function manipulation. IEEE Trans. on Comp., 35(8):677-691, 1986.

2. R. Drechsler and W. Günther. Using lower bounds during dynamic BDD minimization. In Design Automation Conf., pages 29-32, 1999.

3. P. Eades and S. Whitesides. Drawing graphs in two layers. Theoretical Computer Science, 131:361-374, 1994.

4. M. Jünger and P. Mutzel. 2-layer straightline crossing minimization: Performance of exact and heuristic algorithms. Journal of Graph Algorithms and Applications, $1(1): 1-25,1997$.

5. M. Laguna, R. Martí, and V. Valls. Arc crossing minimization in hierarchical digraphs with tabu search. Computers and Operations Research, 24(12):1175-1186, 1997.

6. C. Matuszewski, R. Schönfeld, and P. Molitor. Using sifting for $k$-layer straightline crossing minimization. In Graph Drawing Conference, LNCS 1731, pages 217-224, 1999.

7. K. Mehlhorn and S. Näher. The LEDA Platform of Combinatorial and Geometric Computing. Cambridge University Press, 1999. Project home page at http://www.mpi-sb.mpg.de/LEDA/

8. P. Mutzel, T. Zlegier, S. Naher, D. Alberts, D. Ambras, G. Koch, M. Jünger, C. Buchheim, and S. Leipert. A library of algorithms for graph drawing. In International Symposium on Graph Drawing, LNCS 1547, pages 456-457, 1998. Project home page at http://www.mpi-sb.mpg.de/AGD/

9. S. Panda and F. Somenzi. Who are the variables in your neighborhood. In Int'l Conf. on CAD, pages 74-77, 1995.

10. R. Rudell. Dynamic variable ordering for ordered binary decision diagrams. In Int'l Conf. on CAD, pages 42-47, 1993 . 